Supporting Information

\title{
Optically Stimulated Synaptic Devices Based on the Hybrid Structure of Silicon
}

\section{Nanomembrane and Perovskite}

Lei Yin ${ }^{a}$, Wen Huang ${ }^{a}$, Rulei Xiao ${ }^{b}$, Wenbing Peng ${ }^{a}$, Yiyue Zhu ${ }^{a}$, Yiqiang Zhang ${ }^{a, c}$, Xiaodong Pi ${ }^{a}{ }^{*}$ and Deren Yang ${ }^{a *}$

${ }^{a}$ State Key Laboratory of Silicon Materials and School of Materials Science and Engineering, Zhejiang University, Hangzhou, Zhejiang 310027, China

${ }^{\mathrm{b}}$ National Laboratory of Solid State Microstructures, Collaborative Innovation Center of Advanced Microstructures and College of Engineering and Applied Sciences, Nanjing University, Nanjing 210093, China

${ }^{c}$ School of Materials Science and Engineering, Henan Institute of Advanced Technology, Zhengzhou University, Zhengzhou, Henan 450001, China

*Corresponding authors: Xiaodong Pi (email: xdpi@zju.edu.cn), Deren Yang (email: mseyang@zju.edu.cn) 


\section{Methods}

Device Fabrication: The devices were fabricated by using SOI wafers purchased from Soitec. The thickness of the top $\mathrm{Si} \mathrm{NM}$ and that of the buried oxide $\left(\mathrm{SiO}_{2}\right)$ were $50 \mathrm{~nm}$ and $145 \mathrm{~nm}$, respectively. The top Si NM was doped with boron at the concentration of $10^{15} \mathrm{~cm}^{-3}$, having a resistivity of about $10 \Omega \cdot \mathrm{cm}$. SOI wafers were first cleaned by sonication in deionized water, isopropanol, acetone and ethanol in sequence. After drying by blowing nitrogen gas, they were dipped into a diluted HF solution to remove native oxide. $120 \mathrm{~nm}$ thick gold was then thermally evaporated on Si NM via a shadow mask, leading to $25 \mu \mathrm{m}$ long and $500 \mu \mathrm{m}$ wide channels. Ultraviolet/ozone (UVO) treatment was carried out to improve the surface hydrophilicity of Si NM afterward. A perovskite thin film was subsequently deposited on Si NM by spin-coating a 1.2 M precursor solution, which was a mixture of $\mathrm{CH}_{3} \mathrm{NH}_{3} \mathrm{I} / \mathrm{PbI}_{2}$ in the mole ratio of $1: 1$ in a solution containing dimethyl sulfoxide and $\mathrm{N}, \mathrm{N}$ dimethylformamide with the volume ratio of 1:4. PMMA $\left(\mathrm{Mw}=350 \mathrm{~kg} \mathrm{~mol}^{-1}\right)$ dissolved in anhydrous n-butyl acetate (45 $\mathrm{mg} \mathrm{mL}^{-1}$ ) was finally spin-coated at $800 \mathrm{rpm}$ for $45 \mathrm{~s}$ to cover the perovskite film, giving rise to a complete device structure. The deposition of both the perovskite and PMMA was performed in a glovebox filled with nitrogen gas. In addition, a structure only without the deposition of the perovskite film was used to make Si-NM transistors.

Characterization: The UV-vis spectrum was measured with a UV-vis spectrometer (HITACHI U4100). The X-ray diffraction (XRD) patterns were obtained by using a SHIMADZU LabX XRD6000 diffractometer with $\mathrm{Cu} \mathrm{K \alpha}(\lambda=1.5406 \AA)$ radiation. SEM investigation was carried out on a Hitachi S4800 field emission microscope. UPS measurements were performed by using Thermo Scientific ESCALAB 250Xi with a $21.2 \mathrm{eV}$ He-Ia source. All the devices were measured with a homemade system, ${ }^{22}$ which included a semiconductor parameter analyzer (FS480, PDA Co. Ltd), a 
laser at the wavelength of $532 \mathrm{~nm}$, TTL -controlled optical shutters and an arbitrary function generator (RIGOL DG5100). A power meter (Thorlabs GmbH., PM 100D) was employed to obtain the power density of optical spikes.

Simulation: The optical characteristics of a synaptic transistor was simulated by using a FDTD software (Lumerical Solutions Inc.). The simulation model was based on the cross-sectional SEM images of the synaptic transistor. The cross-sectional area of the device was defined as the $x-z$ plane. The incident light with the electric field intensity of $1 \mathrm{~V} / \mathrm{m}$ was set as a plane wave with the wavelength of $532 \mathrm{~nm}$ and the direction along the $z$-axis. The boundary of the $z$ direction was set as a perfect matching layer, while the boundaries of the $x$ and $y$ directions were both periodic. All the materials were divided into continuous and numerous meshes. The complex refractive index of Si NM was chosen to be the same as that of bulk Si since the thickness of Si NM was not in the quantum confinement regime. ${ }^{1}$ The complex refractive index of $\mathrm{MAPbI}_{3}$ was derived from Löper et al.'s work. ${ }^{2}$ The absorption distribution in a synaptic transistor was simulated by calculating the power absorption per unit volume (Pabs) with the Pabs_adv analysis group of FDTD. The optical power density of absorbed light was normalized by assuming the maximum value as 1 .

Letter recognition was simulated with the assistance of the program of Matlab. An image of the letter "H" was taken as an example. The image was divided into 1960 squares. The image definition increased with the increase of the quantity of the recognized squares. The maximum value of EPSC evoked by 30 optical spikes at $V_{G}=-4 \mathrm{~V}$ was assumed to correspond to all squares being recognized. The number of the recognized squares was obtained by considering the ratio of the real-time EPSC to the maximum EPSC. The higher the EPSC was, the clearer the image would be. 


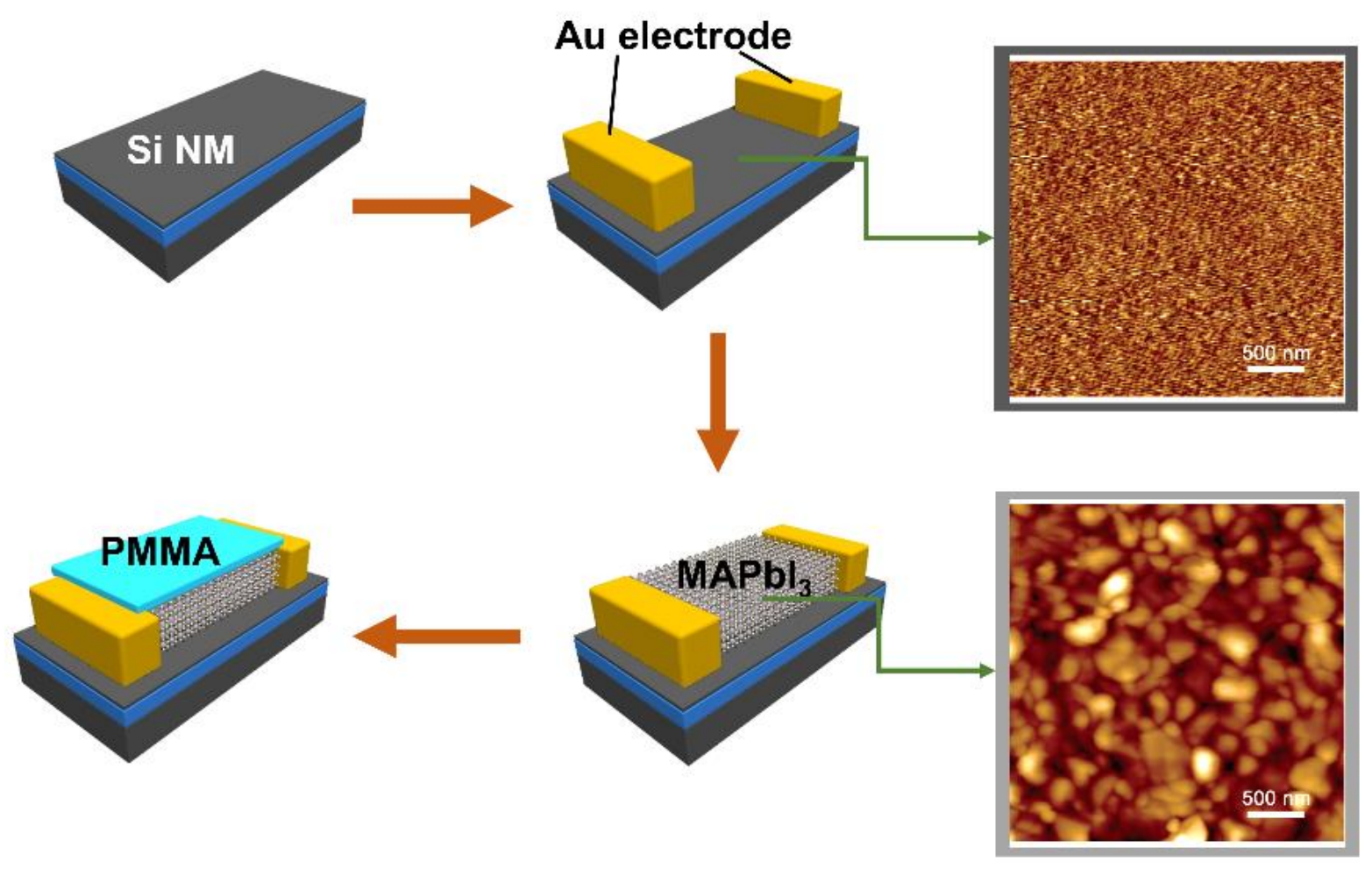

Fig. S1. Process flow of device fabrication with the AFM images of Si NM and $\mathrm{MAPbI}_{3}$. 
Fig. S2. SEM image of the surface of $\mathrm{MAPbI}_{3}$. The inset shows the high-magnification SEM image.

(a)

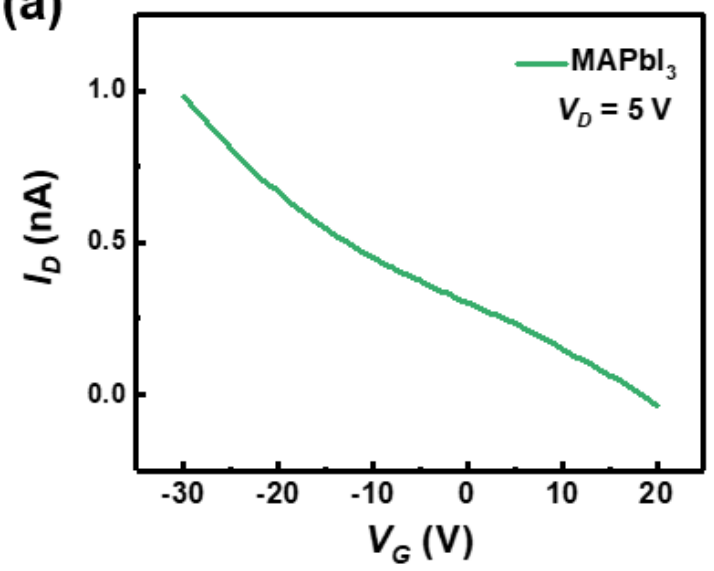

(b)

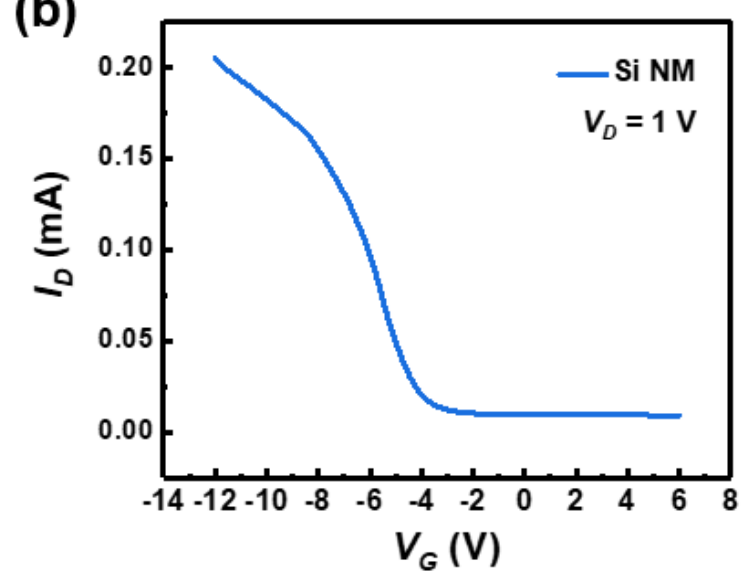

Fig. S3. (a) Transfer curve of a $\mathrm{MAPbI}_{3}$ transistor. $V_{G}$ was swept from 20 to $-30 \mathrm{~V}$ at a fixed $V_{D}$ of $5 \mathrm{~V}$. (b) Transfer curve of a Si-NM transistor. $V_{G}$ was swept from 6 to $-12 \mathrm{~V}$ at a fixed $V_{D}$ of $1 \mathrm{~V}$. The devices are measured in the dark. The $\mathrm{MAPbI}_{3}$ transistor exhibits poor gate tunability, which is due to the ion-screening effect induced by ion migration and accumulation in $\mathrm{MAPbI}_{3}$ (Senanayak et al, Sci. $A d v$., 2017, 3, 1-11). Nevertheless, we manage to obtain that the field-effect mobility of the $\mathrm{MAPbI}_{3}$ transistor is $1.2 \times 10^{-5} \mathrm{~cm}^{2} \mathrm{~V}^{-1} \mathrm{~s}$ ${ }^{1}$ in the linear region. The field-effect mobility of the Si-NM transistor is found to be $45 \mathrm{~cm}^{2} \mathrm{~V}^{-1} \mathrm{~s}^{-1}$ in the linear region. 
(a)

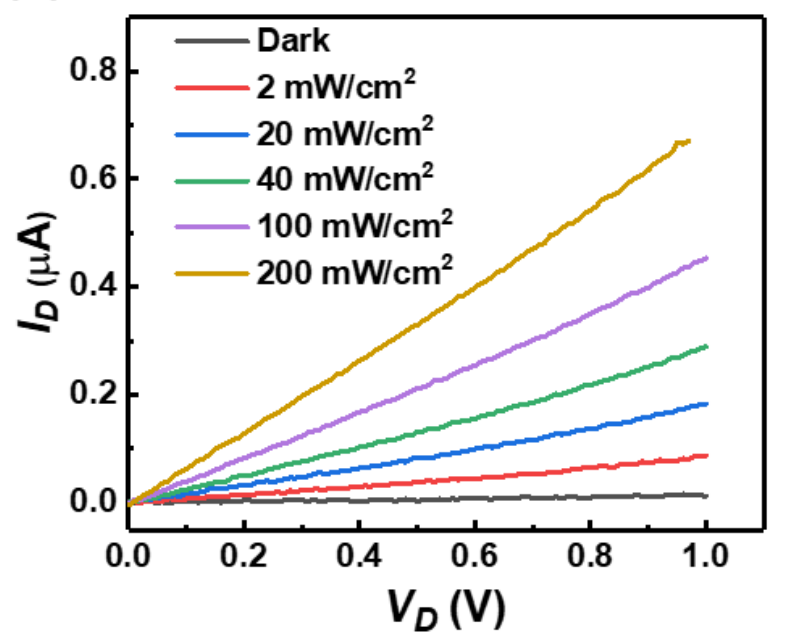

(b)

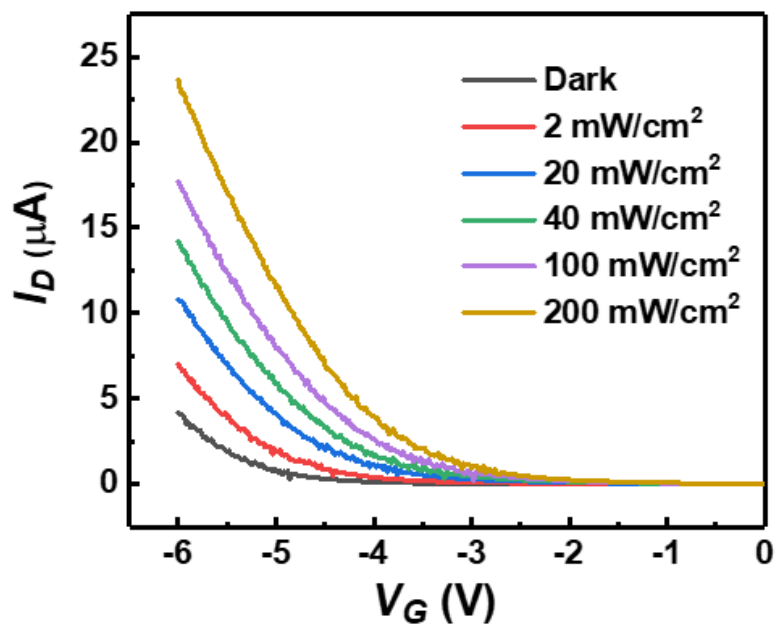

Fig. S4. a) Output characteristics of a synaptic device under the illumination of a $532 \mathrm{~nm}$ laser with various power densities. $V_{D}$ was swept from 0 to $1 \mathrm{~V}$ at a fixed $V_{G}$ of $0 \mathrm{~V}$. b) Transfer characteristics of a synaptic device under the illumination of a $532 \mathrm{~nm}$ laser with various power densities. $V_{G}$ was swept from 0 to $-6 \mathrm{~V}$ at a fixed $V_{D}$ of $0.01 \mathrm{~V}$.

(a)

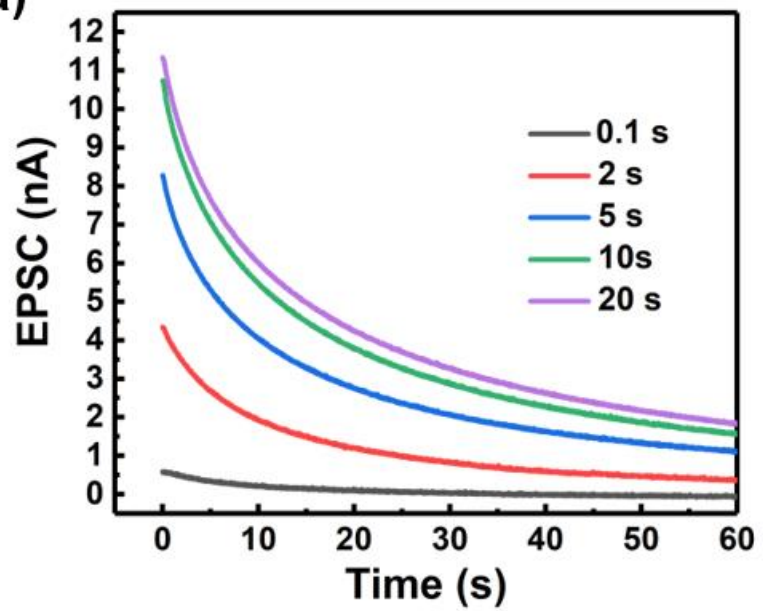

(b)

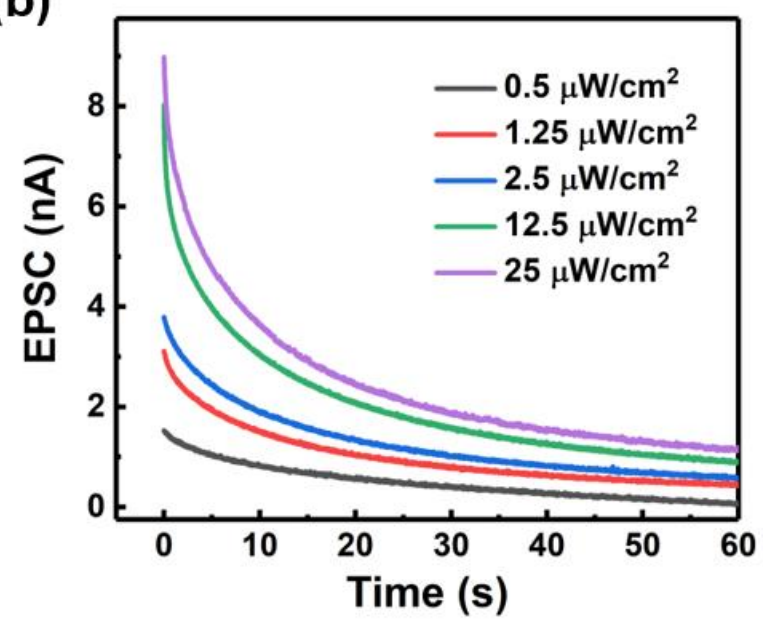

Fig. S5. a) Effect of the duration time of an optical spike on the decay of the EPSC. The wavelength and power density of the optical spike are $532 \mathrm{~nm}$ and $1 \mu \mathrm{W} / \mathrm{cm}^{2}$, respectively. b) Effect of the power intensity of an optical spike on the decay of the EPSC. The wavelength and duration of the optical spike are $532 \mathrm{~nm}$ and $200 \mathrm{~ms}$, respectively. 
(a)

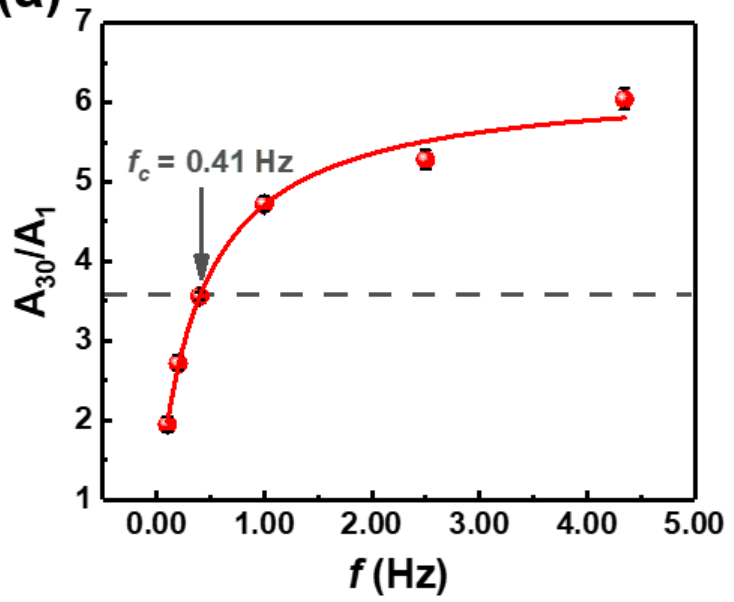

(b)

\section{High-pass filtering}
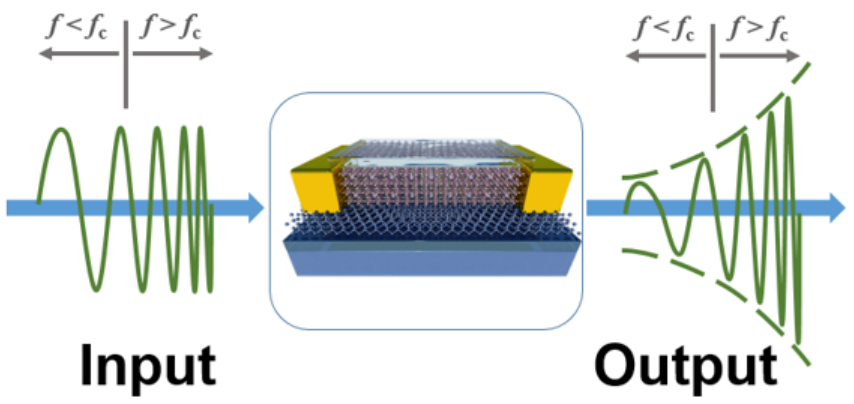

Fig. S6. a) The gain $(\mathrm{g}(f))$ of $\operatorname{EPSC}\left(\mathrm{A}_{30} / \mathrm{A}_{1}\right)$ is fitted with a sigmoidal-shaped function $\mathrm{g}(f)=\left(a_{1}-\right.$ $\left.a_{2}\right) /\left(1+\left(f \mid f_{\mathrm{c}}\right)^{p}\right)+a_{2}$, where $p$ is the order of the function, $f_{\mathrm{c}}$ is the cut-off frequency, $a_{1}$ and $a_{2}$ are the initial and final amplitude. The gain curve can be best fitted with $p=1$. The fitting gives rise to the value of $0.41 \mathrm{~Hz}$ for $f_{\mathrm{c}}$.

b) Schematic illustration of high-pass filtering. The input signal has frequencies varying from low to high. The magnitude of the output signal varies with the frequency of the input signal. For the input signal with the frequency lower than $f_{\mathrm{c}}$ it is weakened at the output. In contrast, it is enhanced at the output if the input signal with the frequency higher than $f_{\mathrm{c}}$. This is the so-called high-pass filtering.

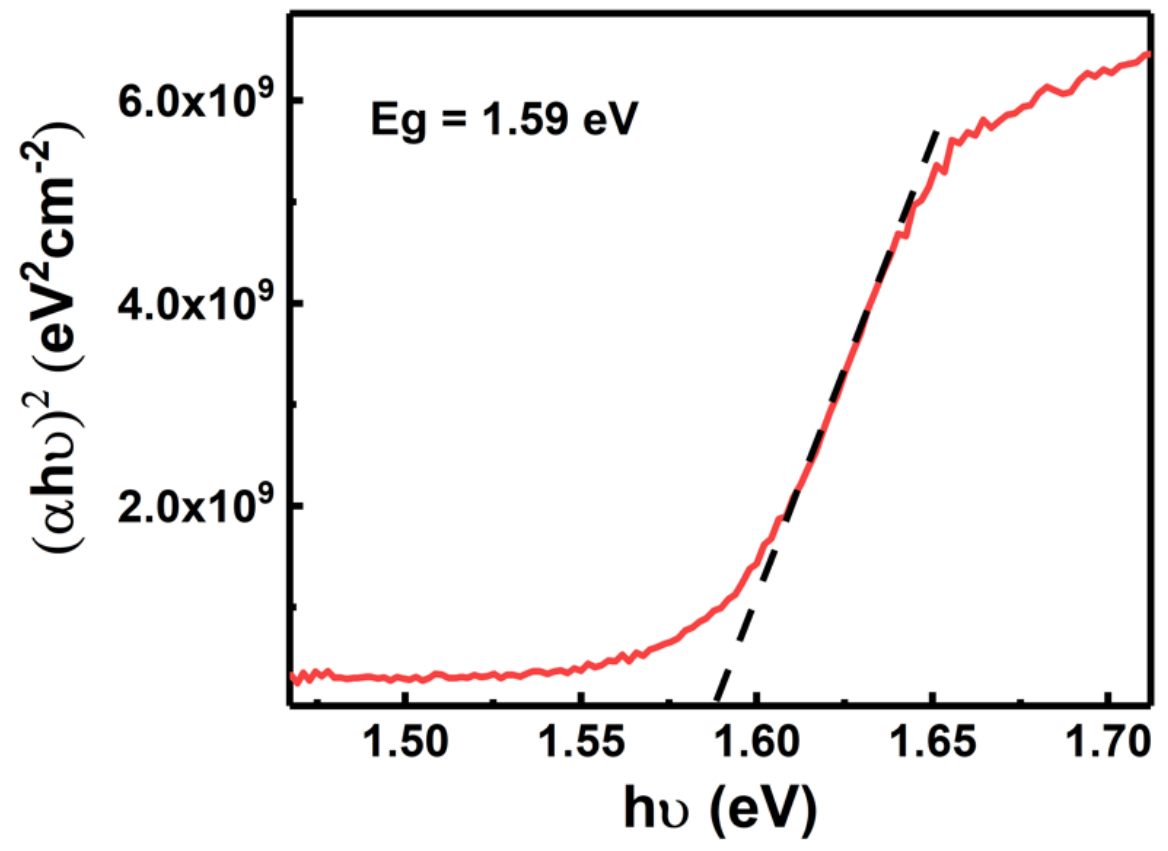

Fig. S7. Tauc plot used to determine the direct bandgap of $\mathrm{MAPbI}_{3}$. 


\section{Working Mechanism}

The behavior of photogenerated carriers in the hybrid structure of $\mathrm{Si} \mathrm{NM}$ and $\mathrm{MAPbI}_{3}$ during the operation of a synaptic transistor at $V_{\mathrm{G}}=0 \mathrm{~V}$ can be depicted by using the energy band diagram in Fig. 4c. An optical spike absorbed by $\mathrm{MAPbI}_{3}$ gives rise to photogenerated holes and electrons. The builtin electric field between $\mathrm{Si} N M$ and $\mathrm{MAPbI}_{3}$ effectively moves the photogenerated holes into $\mathrm{Si} \mathrm{NM}$, leaving the photogenerated electrons in $\mathrm{MAPbI}_{3}$. This means that heterojunction-induced photogating now takes place. ${ }^{3,4}$ As a result of the photogating, the concentration of holes in Si NM increases, leading to the increase of the drain current of the synaptic transistor. Hence, the EPSC is observed with the optical spike. Since photogenerated holes transferred into Si NM are spatially separated from photogenerated electrons remaining in $\mathrm{MAPbI}_{3}$, they have prolonged lifetime. Therefore, we see the slow decay of the EPSC after the optical spike stops.

When the synaptic transistor works at $V_{\mathrm{G}}<0$, the Fermi level moves toward the valence band maximum in Si NW. This causes both the conduction band and valence band of Si NM to more seriously bent at the $\mathrm{Si}-\mathrm{NM} / \mathrm{MAPbI}_{3}$ interface, enhancing the built-in electric field between $\mathrm{Si} \mathrm{NM}$ and $\mathrm{MAPbI}_{3}$ (Fig. 4c). Hence, more significant photogating effect is produced. The resulting more effective extraction of the photogenerated holes of $\mathrm{MAPbI}_{3}$ into $\mathrm{Si} \mathrm{NM}$ explains the increase of the EPSC with the change of the backgate voltage toward the negative direction (Fig. 4a). Additionally, the enhanced built-in electric field in the more significant photogating leads to more effective spatial separation between photogenerated holes transferred into Si NM and photogenerated electrons remaining in $\mathrm{MAPbI}_{3}$. This makes photogenerated holes that have reached $\mathrm{Si} \mathrm{NM}$ have even longer lifetime. Therefore, we see the decay time of the EPSC increases with the change of the backgate voltage toward the negative direction (Fig. S8). 


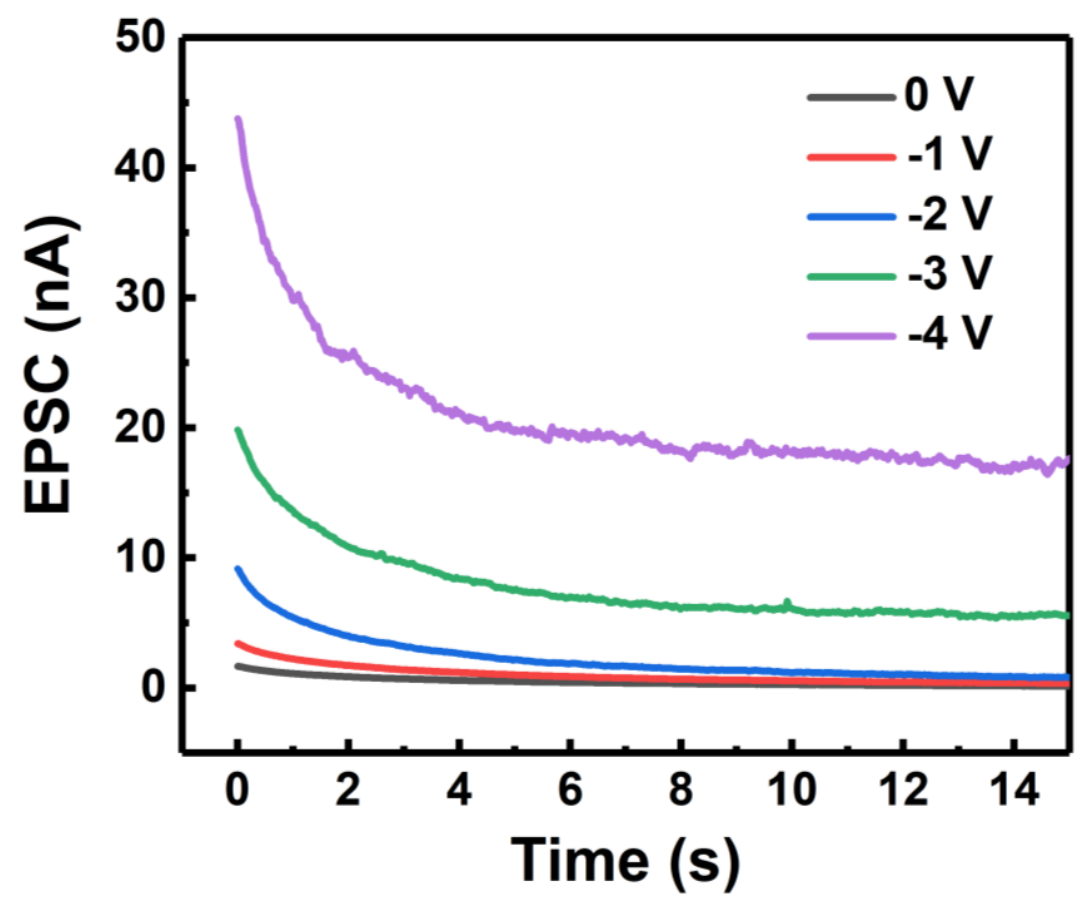

Fig. S8. Effect of the backgate voltage on the decay of the EPSC triggered by an optical spike. When the backgate voltage increases from 0 to $-4 \mathrm{~V}$, the decay time increases by a fact of $\sim 3$. The wavelength, duration and power density of each optical spike are $532 \mathrm{~nm}, 200 \mathrm{~ms}$ and $1 \mu \mathrm{W} / \mathrm{cm}^{2}$, respectively.

Please note that defects such as isolated oxygen vacancies $\left(\mathrm{VO}_{\mathrm{O}}\right)$ may trap electrons in $\mathrm{SiO}_{x}{ }^{5}$ at the surface of Si NM, probably giving rise to photogating as well. ${ }^{3}$ In order to further clarify the working mechanism of our devices, we have compared the threshold-voltage shift and the decay of photocurrent between a $\mathrm{Si}-\mathrm{NM}$ transistor and a $\mathrm{Si}-\mathrm{NM} / \mathrm{MAPbI}_{3}$ transistor under illumination. It is found that the threshold voltage of the Si-NM transistor hardly changes when it is illuminated (Fig. S9a). This means that the ultra-thin $\mathrm{SiO}_{x}$ at the surface of $\mathrm{Si} \mathrm{NM}$ introduces negligible photogating in the current work. In contrast, the threshold voltage of the $\mathrm{Si}-\mathrm{NM} / \mathrm{MAPbI}_{3}$ transistor shifts in the positive direction when it is illuminated (Fig. S9b). The shift of the threshold voltage $\left(\Delta V_{t h}\right)$ increases with the increases of the power density $(P)$ of the illumination. Such a changes can be well fitted by using $\Delta V_{t h}=\alpha P^{\beta}$, where $\alpha$ and $\beta$ are constants (Fig. S9c). The fitting gives rise to the value of 0.31 for $\beta$. Since $\beta$ is smaller than 
(a)
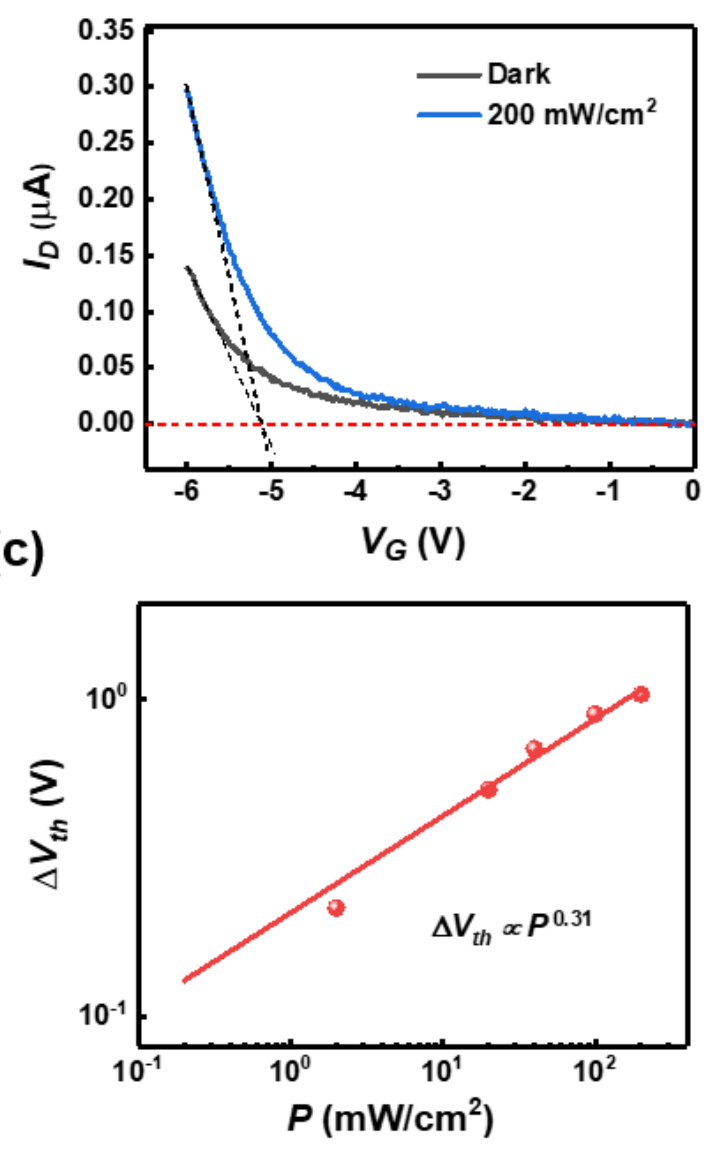

(b)
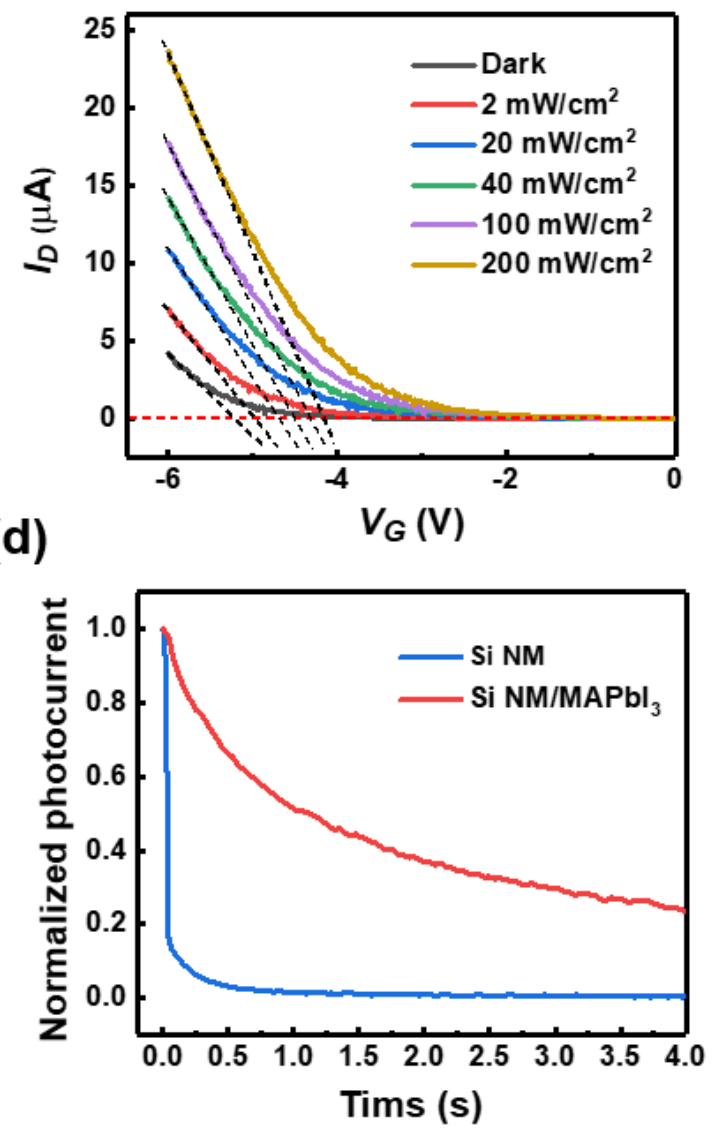

Fig. S9. a) Transfer characteristics of a Si-NM transistor in the dark and under the illumination of a $532 \mathrm{~nm}$ laser with the power density of $200 \mathrm{~mW} / \mathrm{cm}^{2}$. $V_{G}$ was swept from 0 to $-6 \mathrm{~V}$ at a fixed $V_{D}$ of $0.01 \mathrm{~V}$. The threshold voltage $\left(V_{t h}\right)$ is determined by the $x$-intercept point of the tangential line for the linear region of a transfer curve. It is found that the values of $V_{t h}$ are both $\sim-5.12 \mathrm{~V}$ for the Si-NM transistor in the dark and under illumination. b) Transfer characteristics of a $\mathrm{Si}-\mathrm{NM} / \mathrm{MAPbI}_{3}$ transistor in the dark and under the illumination of a $532 \mathrm{~nm}$ laser with varying power density. $V_{G}$ was swept from 0 to $-6 \mathrm{~V}$ at a fixed $V_{D}$ of $0.01 \mathrm{~V}$. $V_{t h}$ is $\sim-5.20 \mathrm{~V}$ for the $\mathrm{Si}-\mathrm{NM} / \mathrm{MAPbI}_{3}$ transistor in the dark. $V_{\text {th }}$ changes from -4.98 to $-4.16 \mathrm{~V}$ when the power density of the illumination increases from 2 to $200 \mathrm{~mW} / \mathrm{cm}^{2}$. c) Dependence of the shift of the threshold voltage $\left(\Delta V_{t h}\right)$ of the $\mathrm{Si}-\mathrm{NM} / \mathrm{MAPbI}_{3}$ transistor on the power density of the illumination. Such a dependence is fitted by using $\Delta V_{t h}$ $=\alpha P^{\beta}$, where $\alpha$ and $\beta$ are constants. The fitting gives rise to the value of 0.31 for $\beta$. d) Normalized photocurrent decay of the $\mathrm{Si}-\mathrm{NM}$ or $\mathrm{Si}-\mathrm{NM} / \mathrm{MAPbI}_{3}$ transistor after the stimulation of an optical spike with the wavelength of $532 \mathrm{~nm}$ and the duration of $200 \mathrm{~ms}$ at $V_{\mathrm{G}}=0 \mathrm{~V}$ and $V_{\mathrm{D}}=1 \mathrm{~V}$. The power density of the optical spike for the $\mathrm{Si}-\mathrm{NM}\left(\mathrm{Si}-\mathrm{NM} / \mathrm{MAPbI}_{3}\right)$ transistor is $6 \mathrm{~mW} / \mathrm{cm}^{2}\left(1 \mu \mathrm{W} / \mathrm{cm}^{2}\right)$. The decay time for the photocurrent of the $\mathrm{Si}-$ $\mathrm{NM}$ transistor is $0.1 \mathrm{~s}$, while that for the photocurrent of the $\mathrm{Si}-\mathrm{NM} / \mathrm{MAPbI}_{3}$ transistor is $12.0 \mathrm{~s}$. 
1, photogating now indeed occurs. ${ }^{6}$ Hence, it is the $\mathrm{Si}-\mathrm{NM} / \mathrm{MAPbI} 3$ heterojunction that introduces the photogating. In addition, we find that the decay time for the photocurrent of the Si-NM transistor is two orders of magnitude smaller than that for the photocurrent of the Si-NM/MAPbI 3 transistor (Fig. S9d). This implies that the ultra-thin $\mathrm{SiO}_{x}$ at the surface of $\mathrm{Si}$ NM plays a negligible role in the present photogating once more. Please note that carrier transport may be also hardly affected by the ultra-thin $\mathrm{SiO}_{x}$ because tunneling readily operates.

(a)

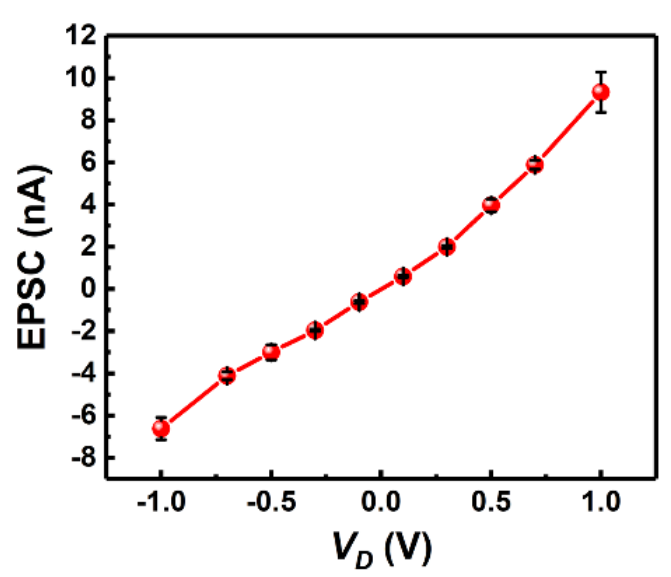

(c)

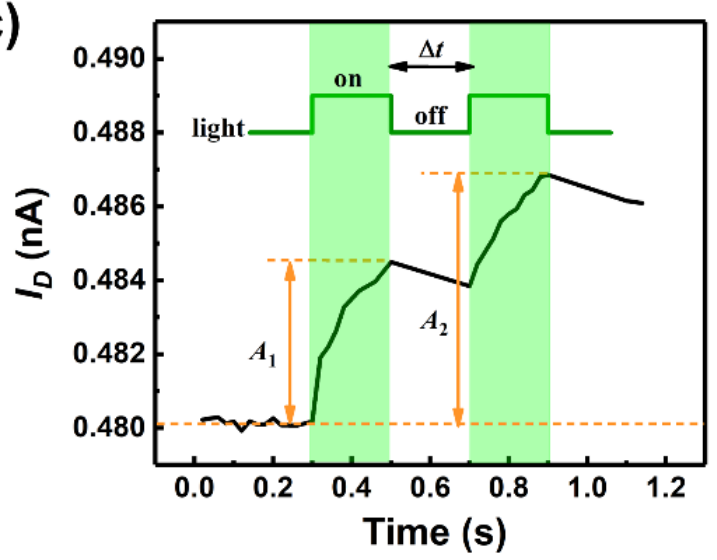

(b)

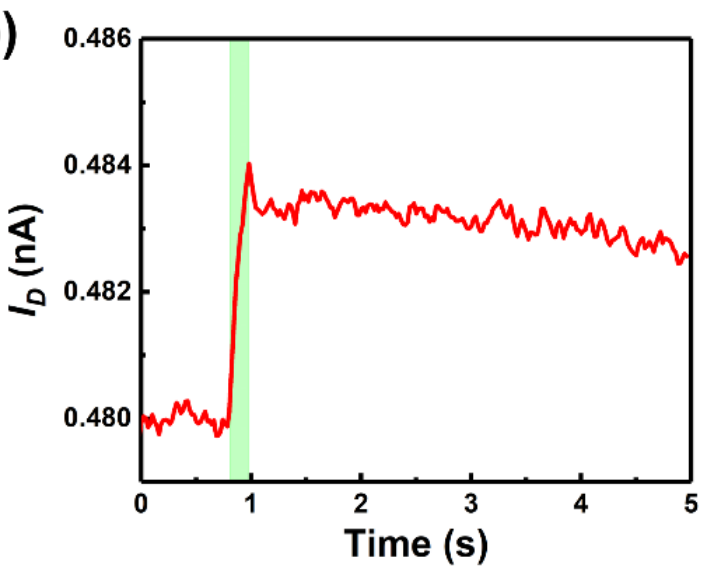

(d)

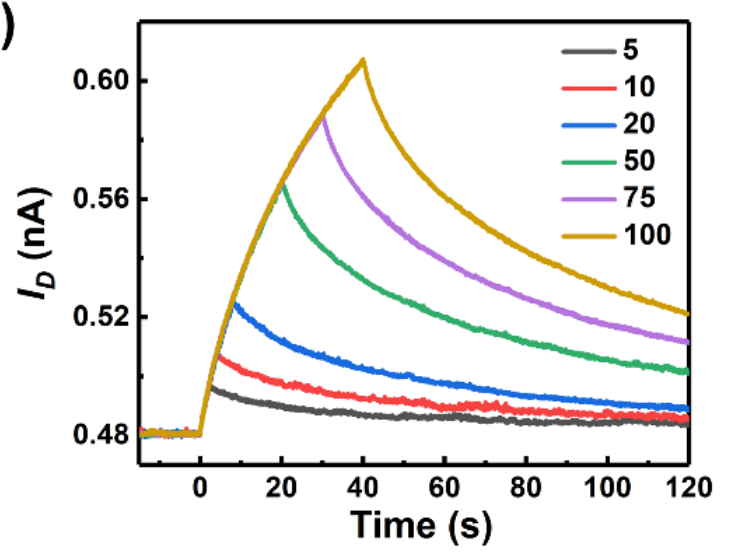

Fig. S10. a) Dependence of the EPSC triggered by an optical spike on the source-drain voltage $\left(V_{\mathrm{D}}\right)$. Error bars represent standard deviation obtained from five independent tests of a synaptic transistor. b) EPSC of a synaptic transistor at $V_{D}=0.01 \mathrm{~V}$ triggered by an optical spike. c) PPF behavior of a synaptic transistor at $V_{D}=0.01 \mathrm{~V}$ triggered by a pair of optical spikes with an interval time ( $\Delta t)$ of $200 \mathrm{~ms}$. d) Transition from STM to LTM for a synaptic transistor at $V_{D}=0.01 \mathrm{~V}$ with the increasing quantity of optical spikes. The wavelength, duration and power density of the optical spike are $532 \mathrm{~nm}, 200 \mathrm{~ms}$ and $1 \mu \mathrm{W} / \mathrm{cm}^{2}$, respectively. 


\section{References}

(1) Chen, F.; Ramayya, E. B.; Euaruksakul, C.; Himpsel, F. J.; Celler, G. K.; Ding, B. J.; Knezevic, I.; Lagally, M. G. Quantum Confinement, Surface Roughness, and the Conduction Band Structure of Ultrathin Silicon Membranes. ACS Nano 2010, 4 (4), 2466-2474. https://doi.org/Doi 10.1021/Nn100275z.

(2) Löper, P.; Stuckelberger, M.; Niesen, B.; Werner, J.; Filipič, M.; Moon, S. J.; Yum, J. H.; Topič, M.; De Wolf, S.; Ballif, C. Complex Refractive Index Spectra of CH3NH3PbI3 Perovskite Thin Films Determined by Spectroscopic Ellipsometry and Spectrophotometry. J. Phys. Chem. Lett. 2015, 6 (1), 66-71. https://doi.org/10.1021/jz502471h.

(3) Fang, H.; Hu, W. Photogating in Low Dimensional Photodetectors. Adv. Sci. 2017, 4 (12), 1700323. https://doi.org/10.1002/advs.201700323.

(4) Xie, C.; You, P.; Liu, Z.; Li, L.; Yan, F. Ultrasensitive Broadband Phototransistors Based on Perovskite/Organic-Semiconductor Vertical Heterojunctions. Light Sci. Appl. 2017, 6 (8), e17023. https://doi.org/10.1038/1sa.2017.23.

(5) Liu, Y.; Stradins, P.; Deng, H.; Luo, J.; Wei, S. H. Suppress Carrier Recombination by Introducing Defects: The Case of Si Solar Cell. Appl. Phys. Lett. 2016, 108 (2), 13-17. https://doi.org/10.1063/1.4939628.

(6) Takanashi, Y.; Takahata, K.; Muramoto, Y. Characteristics of InAlAs/InGaAs High-ElectronMobility Transistors under Illumination with Modulated Light. IEEE Trans. Electron Devices 1999, 46 (12), 2271-2277. https://doi.org/10.1109/16.808049. 